PROCEEDINGS OF THE

AMERICAN MATHEMATICAL SOCIETY

Volume 126, Number 12, December 1998, Pages 3767-3768

S 0002-9939(98)05066-7

\title{
A REMARK ON MANSFIELD'S IMPRIMITIVITY THEOREM
}

\author{
CHI-KEUNG NG
}

(Communicated by David R. Larson)

\begin{abstract}
We show that the Morita equivalence part of Mansfield's Imprimitivity Theory can be obtained by Green's Imprimitivity Theorem (and duality theory).
\end{abstract}

In [5], Mansfield developed a very interesting theory of induced representations for group coactions. He started from very basic things and developed all the machineries that he needed.

In this paper, we will reconstruct part of his theory (namely, the Morita equivalence part) using Green's Imprimitivity Theorem and the recently developed theory of group coactions and their crossed products. For basic definitions and properties of group coactions, we refer the readers to [6] and [8].

Our simple approach is based on Raeburn's reformulation (see [8, 2.9(3)]) of Green's Imprimitivity Theorem $([2$, Theorem 6$])$ : if $B$ is a $C^{*}$-algebra with an action $\alpha$ by a locally compact group $G$ and $\hat{\alpha}$ is the dual coaction on $B \times{ }_{\alpha} G$, then $B \times{ }_{\alpha \mid} H$ is strongly Morita equivalent to $\left(B \times_{\alpha} G\right) \times_{\hat{\alpha} \mid}(G / H)$ (where $\alpha \mid$ and $\hat{\alpha} \mid$ are the restrictions of $\alpha$ and $\hat{\alpha}$ on $H$ and $G / H$ respectively).

It is well known that given a locally compact group $G$ and a closed normal subgroup $H$, there is a quotient map $Q_{H}$ from $C^{*}(G)$ to $C^{*}(G / H)$. The spatial version of this fact is the only thing that we need from [5] for our consideration (see [5, Lemmas 3 and 4]): if $H$ is a closed normal amenable subgroup of $G$, then there exists a *-homomorphism $q_{H}$ from $C_{r}^{*}(G)$ to $C_{r}^{*}(G / H)$ (which preserves comultiplications) such that for any reduced coaction $\epsilon$ of $G$ on a $C^{*}$-algebra $A$, $\epsilon \mid=\left(i d \otimes q_{H}\right) \circ \epsilon$ is a reduced coaction on $A$ by $G / H$.

Lemma 1.1. Let $D$ be a $C^{*}$-algebra with a full coaction $\delta$ by a locally compact group $G$. Let $I=\operatorname{Ker}\left(\left(i d \otimes \lambda_{G}\right) \circ \delta\right)$, and let $\epsilon$ be the reduced coaction on the quotient $D / I$ of $D$ induced by $\delta$ (see [8, Section 3]). If $H$ is a closed normal amenable subgroup of $G$, then $D \times_{\delta \mid}(G / H)=(D / I) \times_{\epsilon \mid}(G / H)$.

Proof. Let $\epsilon_{1}=\left(i d \otimes \lambda_{G / H}\right) \circ(\delta \mid)$ (where $\lambda_{G / H}$ is the left regular representation of $G / H)$. Then, by $[6,2.16], D \times_{\delta \mid}(G / H)=D \times_{\epsilon_{1}}(G / H)$ (note that the amenability condition of $[6,2.16]$ is automatically satisfied in the case of coactions of locally compact groups). Let $J=\operatorname{Ker}\left(\epsilon_{1}\right)=\operatorname{Ker}\left(\left(i d \otimes \lambda_{G / H}\right) \circ\left(i d \otimes Q_{H}\right) \circ \delta\right)$. Since $\lambda_{G / H} \circ Q_{H}=q_{H} \circ \lambda_{G}, J=\operatorname{Ker}\left(\left(i d \otimes q_{H}\right) \circ\left(i d \otimes \lambda_{G}\right) \circ \delta\right) \supseteq I$. Moreover, $(\epsilon \mid) \circ Q=$

Received by the editors June 20, 1997.

1991 Mathematics Subject Classification. Primary 46L55.

Key words and phrases. Coactions, crossed products, Imprimitivity Theorem, Morita equivalence. 
$\left(i d \otimes q_{H}\right) \circ \epsilon \circ Q=\left(i d \otimes q_{H}\right) \circ(Q \otimes i d) \circ\left(i d \otimes \lambda_{G}\right) \circ \delta=(Q \otimes i d) \circ \epsilon_{1}$ (where $Q$ is the quotient map from $D$ to $D / I)$. Hence by $[6,2.18],(D / I) \times_{\epsilon \mid}(G / H)=D \times_{\epsilon_{1}}(G / H)$.

We can now prove the following Imprimitivity Theorem for coactions (note that coactions considered in section 5 of [5] are non-degenerate).

Theorem 1.2. Let $A$ be a $C^{*}$-algebra with a non-degenerate reduced coaction $\epsilon$ by a locally compact group $G$. Let $H$ be a closed normal amenable subgroup of $G$. Then $\left(A \times{ }_{\epsilon} G\right) \times_{\hat{\epsilon} \mid} H$ is strongly Morita equivalent to $A \times_{\epsilon \mid}(G / H)$.

Proof. Let $B=A \times_{\epsilon} G$, and let $\alpha$ be the dual action of $\epsilon$. Let $\delta$ and $\delta_{r}$ be respectively the full and the reduced dual coactions of $\alpha$ (note that $\delta$ equals $\hat{\alpha}$ in the above notation). Then by Green's Theorem, we only need to show that $\left(B \times_{\alpha} G\right) \times_{\delta \mid}(G / H)$ is strongly Morita equivalent to $A \times_{\epsilon \mid}(G / H)$. If we put $D=B \times{ }_{\alpha} G$ into Lemma 1.1, then the coaction $\epsilon$ in Lemma 1.1 equals $\delta_{r}$ (see [8, $3.2(1)])$ and so $\left(B \times_{\alpha} G\right) \times_{\delta \mid}(G / H)=\left(B \times_{\alpha, r} G\right) \times_{\delta_{r} \mid}(G / H)$. Now since $\delta_{r}$ is the bidual coaction on $B \times_{\alpha, r} G=A \otimes \mathcal{K}\left(L^{2}(G)\right.$ ) (see [3, Theorem 8] or [8, 5.1]), $\delta_{r}=$ $A d(v) \circ(\epsilon \otimes i)$ where $i$ is the trivial coaction on $\mathcal{K}\left(L^{2}(G)\right)$ by the trivial group and $v$ is an $(\epsilon \otimes i)$-cocycle (see [4, 2.7]). Therefore, $\delta_{r} \mid=\left(i d \otimes q_{H}\right) \circ \delta_{r}=A d\left(v^{\prime}\right) \circ((\epsilon \mid) \otimes i)$ where $v^{\prime}=\left(i d \otimes q_{H}\right)(v)$ is an $((\epsilon \mid) \otimes i)$-cocycle. Hence by $[4,2.9]$ and $[6,3.3]$ (or [7, 1.16]), $\left(B \times_{\alpha, r} G\right) \times_{\delta_{r} \mid}(G / H) \cong\left(A \times_{\epsilon \mid}(G / H)\right) \otimes \mathcal{K}\left(L^{2}(G)\right)$ which is clearly strongly Morita equivalent to $A \times_{\epsilon \mid}(G / H)$.

\section{REFERENCES}

[1] S. Baaj and G. Skandalis, $C^{*}$-algèbres de Hopf et théorie de Kasparov équivariante, $K$-theory 2 (1989), 683-721. MR 90j:46061

[2] P. Green, The local structure of twisted covariance algebras, Acta. Math. 140 (1978), 191-250. MR 58:12376

[3] Y. Katayama, Takesaki's duality for a nondegenerate coaction, Math. Scand. 55 (1984), 141151. MR 86b:46112

[4] M. B. Landstad, J. Phillips, I. Raeburn and C. E. Sutherland, Representations of crossed products by coactions and principal bundles, Trans.Amer. Math. Society, Vol 299 (1987), No. 2, 747-784. MR 88f: 46127

[5] K. Mansfield, Induced representations of crossed products by coactions, J. Funct. Anal., 97 (1991), no. 1, 112-161. MR 92h:46095

[6] C. K. Ng, Coactions and crossed products of Hopf $C^{*}$-algebras, Proc. London Math. Soc. (3) 72 (1996), 638-656. MR 97d:46083

[7] J. Quigg, Full and reduced $C^{*}$-coactions, Math. Proc. Camb. Phil. Soc. 116 (1994), 425-450. MR 95g:46126

[8] I. Raeburn, On crossed products by coactions and their representation theory, Proc. London Math. Soc. (3), 64 (1992), 625-652. MR 93e:46080

[9] M. A. Rieffel, Induced representations of $C^{*}$-algebras, Adv. in Math. 13 (1974), 176-257. MR 50:5489

[10] M. A. Rieffel, Strong Morita equivalence of certain transformation group $C^{*}$-algebras, Math. Ann. 222 (1976), 7-22. MR 54:7695

Mathematical Institute, Oxford University, 24-29 St. Giles, Oxford OX1 3LB, United KINGDOM

E-mail address: ng@maths.ox.ac.uk 